\title{
Evaluation of neutron induced reaction cross sections in the resolved and unresolved resonance region at EC - JRC - IRMM
}

\author{
S. Kopecky ${ }^{1}$, F. Emiliani ${ }^{1}$, K. Kauwenberghs ${ }^{1}$, C. Lampoudis ${ }^{1}$, C. Massimi ${ }^{2}$, \\ P.Schillebeeckx ${ }^{1}$, I. Sirakov ${ }^{3}$ and K. Volev ${ }^{1}$ \\ ${ }^{1}$ European Commission, Joint Research Centre, Institute for Reference Materials and \\ Measurements, Retieseweg 111, B-2440 Geel, Belgium \\ ${ }^{2}$ Department of Physics, University of Bologna and sezione INFN of Bologna, Via Irnerio \\ 46, Bologna, 40126 Italy \\ ${ }^{3}$ Institute for Nuclear Research and Nuclear Energy, Sofia, Bulgaria
}

\begin{abstract}
Recent efforts made at the EC-JRC-IRMM to produce evaluated cross section data files for neutron induced reactions are described as well as the methodology applied in both the resolved and unresolved resonance. For the resolved resonance region the paper focuses on a recent evaluation of isotopes present in natural cadmium. For the unresolved resonance region results for gold are presented.
\end{abstract}

\section{Introduction}

Recently the IRMM started to create evaluated cross section data files or neutron induced reactions. A methodology was established with a strong emphasis on the use of all the experimental data reported and described in literature.

For the resolved resonance region (RRR), resonance parameter data (including area data), coherent scattering lengths and thermal cross section data are considered, in addition to well documented experimental time-of-flight (TOF) data available in EXFOR. All the available data are used to define a starting file using the most complete data set as a reference. The reference file is complemented by other data after applying correction factors which are mostly derived from ratios of resonance parameters (energies, $\mathrm{g} \Gamma_{\mathrm{n}}$, capture Kernels ...). In addition, results of deterministic spin and parity assignment measurements are included. If results of optical model calculations are available, the scattering radii at zero energy are derived from these results. Bound states are included to match the scattering and reaction cross sections at thermal energy. Then this generated file is validated and improved using TOF-data available in EXFOR and results of additional cross section experiments at GELINA [1, 2], mostly performed on natural samples. This step, which is based on a resonance shape analysis using the REFIT code [3], is also used to solve discrepancies between existing data, to fill gaps in the available data set and to identify if additional measurements, e.g. on enriched samples, are required. 
In the unresolved resonance region transmission and capture cross section measurements are performed at GELINA following the measurement, data reduction and data analysis procedures as described in Ref. [4].These data are then combined with all available data in literature and finally average total and capture cross sections are parameterized by average resonance parameters applying the Hauser-Feshbach statistical reaction theory [5].

\section{Experiments at GELINA}

The TOF-facility GELINA $[1,2]$ is based on an electron linear accelerator, where electrons are accelerated up to $140 \mathrm{MeV}$. The electron pulses have a duration of $1 \mathrm{~ns}$ [6] and a repetition rate between $50 \mathrm{~Hz}$ and $800 \mathrm{~Hz}$. The electrons are stopped in a mercury cooled rotary target, consisting of depleted uranium and bremsstrahlung is produced. The dominant neutron production mechanisms are $(\gamma, n)$ and $(\gamma, 2 n)$ photo-neutron reactions in the giant dipole resonance. The neutrons are moderated in two $4 \mathrm{~cm}$ beryllium canned water containers mounted on top and bottom of the neutron production target. Depending on the collimation either a fast or a thermalized spectrum can be used for experiments. $\mathrm{BF}_{3}$ proportional counters, placed at different locations around the target hall, are used to monitor the stability of the accelerator and to normalize TOF-spectra to the same total neutron intensity.

Two setups are used for the transmission measurements, one with the detector located at $50 \mathrm{~m}$ the other one at $25 \mathrm{~m}$. In both cases the sample is inserted approximately half way between the neutron source and detector into the beam. Close to the sample positions black resonance filters are put into the neutron beam to estimate the background. The neutron detectors are Li-glass scintillators with a thicknesses of $1.26 \mathrm{~cm}$ at $25 \mathrm{~m}$ of $0.625 \mathrm{~cm}$ at $50 \mathrm{~m}$. During the measurements the sample-in and sample-out positions are cycled approximately every 20 minutes. This is done to reduce the impact of a possible change in machine output or drift of the monitors and neutron detectors. With such a procedure the uncertainty of the normalization of the transmission measurement is smaller than $0.5 \%$.

Capture measurements can be performed at $12.5 \mathrm{~m}, 30 \mathrm{~m}$ or $60 \mathrm{~m}$ station. The prompt $\gamma$-rays emerging from a capture event are detected by $\mathrm{C}_{6} \mathrm{D}_{6}$ liquid scintillators. Each scintillator is coupled directly to a boron-free quartz windowed photo-multiplier tube. At the $12.5 \mathrm{~m}$ and $30 \mathrm{~m}$ station 2 detectors are used. At the $60 \mathrm{~m}$ station the detection system consists of $4 \mathrm{C}_{6} \mathrm{D}_{6}$ detectors. All detectors are mounted at 125 degrees with respect to the direction of the neutron beam. With such a setup, systematic effects caused by the anisotropy of the gamma emission can be minimized. The total energy detection principle together with a pulse height weighting technique is used to determine the capture yield [7]. The stability and resolution of the detector is monitored weekly. The shape of the neutron spectrum is measured simultaneously with a ${ }^{10} \mathrm{~B}$ Frisch gridded ionisation chamber. For higher energies a parallel plate chamber loaded with ${ }^{235} \mathrm{U}$ is used. The chamber is located approximately $1 \mathrm{~m}$ before the capture sample.

For the data reduction the AGS-package [8] is used. This package includes the most important spectra manipulations and performs a full uncertainty propagation accounting for both correlated and uncorrelated uncertainty components.

\section{$3 \mathrm{Cd}-$ the resolved resonance region}

The interest in cadmium is triggered by a very strong resonance at $0.178 \mathrm{eV}$. This resonance was the first ascribed to ${ }^{113} \mathrm{Cd}$ in 1946 by Moyer et al. [9]. As a $1 \mathrm{~mm}$ thick natural cadmium sheet absorbs all neutrons below $0.25 \mathrm{eV}$ and only a small fraction above $0.5 \mathrm{eV}$, it is frequently used as an absorber in resonance integral measurements. To interpret 
and analyze such measurements correctly, an accurate description of the total cross section for neutron induced reactions in ${ }^{\text {nat }} \mathrm{Cd}$ at energies above $0.5 \mathrm{eV}$ is essential. Unfortunately, the total, capture and scattering cross sections in cadmium are not known with sufficient accuracy. Most of the experiments related to reaction cross section measurements on cadmium reported in the literature focus on more fundamental studies, e.g. parity violation, and single particle effects. The experimental observables, i.e. transmissions or capture yields, have never been used in a combined resonance shape analysis to evaluate resonance parameters. In fact, the resonance parameters used for the evaluations are primarily the result of a compilation of resonance parameters available in the literature. Furthermore there has been no consistent, simultaneous evaluation for all the isotopes. This might be the reason for some of the longstanding discrepancies between quantities measured in integral measurements and calculations using the tabulated resonance parameters. To provide data for a more consistent evaluation for all the cadmium isotopes transmission and capture experiments with numerous samples have been carried out at GELINA. An extensive study of the parameters reported in the literature combined with the resonance shape analysis of new experimental data together and well documented TOF-data available derived from EXFOR, were used as a basis to produce an ENDF-6 compatible evaluation for cadmium in the RRR.

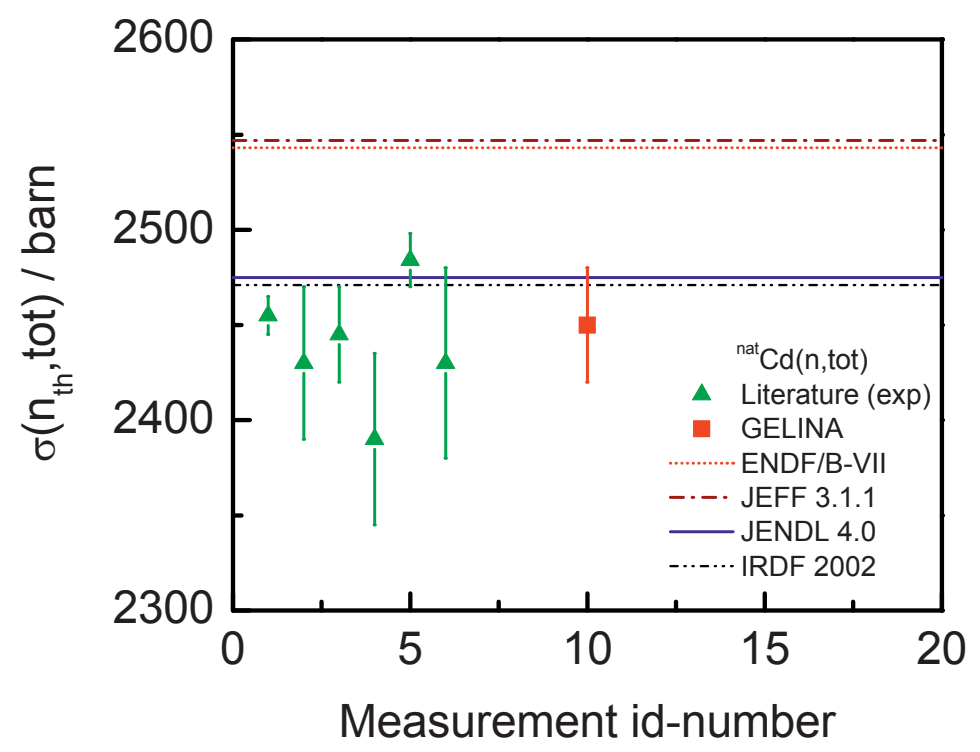

Fig. 1: A comparison of experimental and evaluated data for the thermal total cross section of ${ }^{\text {nat }} \mathrm{Cd}$.

Resonance parameters have been adjusted from thermal up to $10 \mathrm{keV}$. The scattering radii have been adjusted such that the contribution of the resonance scattering and scattering radius match the coherent scattering length given in literature. Results of the transmission measurements with the $25 \mathrm{~mm}$ thick sample were used to make final adjustments of the parameters influencing the scattering cross section. The resulting thermal total cross is in agreement with measurement using thermal neutron beams, but differs from values used in recent evaluations as shown in Fig.1. This study also revealed that further improvement of the cadmium files might be achieved if measurements on enriched ${ }^{114} \mathrm{Cd}$ were performed. Details of all the experiments, the evaluation procedure and results can be found in Volev et al. [10]. 


\section{$4 \mathrm{Au}$ - the unresolved resonance region}

Gold is an extremely important material in nuclear applications. The neutron capture cross section of ${ }^{197} \mathrm{Au}$ is used as a measurement standard to determine neutron induced capture cross sections. It is also proposed as a standard for high energy neutron dosimetry. In addition, cross sections of neutron induced reactions on gold are considered as a test-case for many nuclear reaction model codes. The current evaluation of the capture cross section of ${ }^{197} \mathrm{Au}$ is the result of a project organized by the IAEA, in which a large set of experimental data between $2.5 \mathrm{keV}$ and $2.5 \mathrm{MeV}$ has been used [11]. In the region above $200 \mathrm{keV}$, the neutron induced capture cross section is recommended as a standard for neutron reaction cross section measurements [11]. Below $200 \mathrm{keV}$, due to strong fluctuations caused by the resonance structure of the capture cross section of gold, the data are not recommended as a standard. Nevertheless the cross section is used as a reference for a variety of cross sections measured for astrophysical applications. Unfortunately the standard cross section differs by 6-8 \% from the cross section adopted as astrophysical reference. This latter value is based on a measurement of the energy dependent cross section by Macklin [12] normalized to the results of activation measurements done by Ratzinsky and Kaeppler [13]. However, the results of a measurement by Borella et al. [14], which were not included in the standard evaluation, agree within $2-3 \%$ with the standard evaluation and disagree with the astrophysical reference value, as illustrated in Fig. 2. This finding has been confirmed by recent measurements [15]. In an effort to resolve the differences additional transmission and capture measurements have been performed at GELINA. Efforts have been devoted to reduce systematic bias effects caused by dead-time, background, $\gamma$-ray attenuation in the sample, self-shielding, multiple interaction events, normalization and shape of the neutron flux. To produce reliable correction factors for some of these effects, i.e. self-shielding, multiple interaction events, $\gamma$-ray attenuation in the sample, accurate total cross section data in the URR are required. The present evaluation of the total cross section is based on the measurement by Seth et al. [16], but seems to be in contradiction with results of other experiments (e.g. Purtov et al. [17]) and the total cross section resulting from a dispersive coupled optical model potential. Unfortunately the spread of the experimental data in the unresolved resonance region are rather large.

To improve the evaluation of both the total and capture cross of ${ }^{197} \mathrm{Au}$ in the unresolved resonance region additional transmission and capture measurements are required. Therefore, capture cross section measurements have been carried out at the 12.5 $\mathrm{m}$ capture station and transmission measurements at the $50 \mathrm{~m}$ station of GELINA. Additional transmission measurements are planned at the nELBE facility [18] to bridge the gap between the unresolved resonance region and the continuum. A preliminary analysis of the capture data reveals that average capture cross sections between $5 \mathrm{keV}$ and $80 \mathrm{keV}$ can be deduced with uncertainties below $2 \%$. The results of the total and capture cross section data will be used to produce a full ENDF-6 compatible evaluated data file including full covariance information resulting from a parameterisation of the cross section data using the Hauser-Feshbach as described in Ref. [4] for ${ }^{232} \mathrm{Th}$.

\section{Acknowledgments}

This work was partly supported by the European Commission through the projects EFNUDAT (FP6-036434) and ERINDA (FP7-269499). This work was supported by the 
European Commission within the Seventh Framework Programme through EUFRAT (EURATOM contract no. FP7-211499).

\section{References}

1. W. Mondelaers and P. Schillebeeckx, Notiziario, 11, 19 (2006)

2. M. Flaska, A. Borella, D. Lathouwers, L.C. Mihailescu, W. Mondelaers, A.J.M. Plompen, H. van Dam, T.H.J.J. van der Hagen, Nucl. Instr. Meth. A 531, 394 (2004)

3. M.C. Moxon and J.B. Brisland, "GEEL REFIT, A least squares fitting program for resonance analysis of neutron transmission and capture data computer code", AEAInTec-0630, AEA Technology, October 1991

4. P. Schillebeeckx, B. Becker, Y. Danon et al., accepted for publication in Nuclear Data Sheets

5. I. Sirakov, R. Capote, F. Gunsing, P. Schillebeeckx and A. Trkov, Ann. Nucl. En. 35, 1223 (2008).

6. D. Tronc, J. M. Salomé, K.H. Böckhoff, Nucl. Istrum. Meth. A 228, 217 (1985)

7. A. Borella, G. Aerts, F. Gunsing, M. Moxon, P. Schillebeeckx and R. Wynants, Nucl. Instr. Meth. A 577, 626 (2007)

8. B. Becker et al., submitted to JINST

9. B.J. Moyer, B. Peters and F.H. Schmidt, Phys. Rev. 66, 666 (1946)

10. K. Volev, A. Borella, S. Kopecky, C. Lampoudis, C. Massimi, A. Moens, M. Moxon, P. Schillebeeckx, P. Siegler, I. Sirakov, A. Trkov and R. Wynants, submitted to Nucl. Istrum. Meth. B

11. A.D. Carlson, V.G. Pronyaev, D. L. Smith, N. M. Larson, Z. Chen, G. M. Hale, F.-J. Hambsch, E. V. Gai, Soo-Youl Oh, S. A. Badikov, T. Kawano, H. M. Hofman, H. Vonach and S. Tagesen, Nuclear Data Sheets 110, 3215 (2009)

12. R.L. Macklin, Nucl. Sci. Eng. 79, (1981) 265

13. W. Ratynski and F Käppeler, Phys. Rev C 37, 595 (1988)

14. A. Borella, K. Volev, A. Brusegan, P. Schillebeeckx, F. Corvi, N. Koyumdjieva, N. Janeva, A.A. Lukyanov, Nucl. Sci. Eng. 152, 1 (2006)

15. G. Feinberg, M. Friedmann, A. Kràsa, A. Shor, Y.Eisen, D. Berkovits,D. Cohen, G. gioginis, T. Hirsh, M. Paul, A.J.M. Plompen and E.Tsuk, Phys. Rev C 85, 055810 (2012)

16. K.K. Seth, Phys. Lett 16, 306 (1965)

17. OP.A. Purtov, L.L. Litvinskii, A. Z. Murzin and G.M. Novoselov, At. Energy, 77, 536 (1994)

18. J. Klug, E. Altstadt, C. Beckert, R. Beyer, D. Freiesleben, V. Galindo, E. Grosse, A.R. Junghans, D. Legrady, B. Naumann, K. Noack, G. Rusev, K.D. Schilling, R. Schlenk, R. Schneider, A. Wagner, and F.-P. Weiss, Nucl. Inst. Meth. A 577, 641 (2007) 